\title{
UNIQUE FACTORIZATION IN PARTIALLY ORDERED SETS
}

\author{
JORGE MARTINEZ
}

\begin{abstract}
AbSTRACr. Call an ideal of a lattice $L$ regular if it is maximal with respect to not containing some element of $L$. If the ideal $M$ is maximal without $x \in L$ we say $M$ is a value of $x$. A special idea! $K$ is a regular ideal which is maximal without some element having only one value (namely $K$ ). Our main theorem is that the following three statements are equivalent: (1) The lattice of ideals of the lattice $L$ is completely distributive. (2) Each regular ideal is special. (3) $L$ is distributive, and each element $x \in L$ can be written uniquely as a finite join of pairwise incomparable, finitely join irreducible elements of $L$.

By carefully generalizing our notions to partially ordered sets we get a similar theorem in this context.
\end{abstract}

1. The main objective of this paper is to present a characterization of those lattices $L$ in which every element can be expressed as a finite join of finitely join irreducible elements, in terms of properties of the lattice of ideals of $L$. By carefully adapting our definitions to partially ordered sets (henceforth: p.o. sets) we can obtain a more general result. We proceed in the next few paragraphs to define the pertinent concepts and state the main theorem in detail.

An ideal of a p.o. set $P$ is a subset $M$ having the following properties: (1) if $a \in M$ and $b \leqq a$ then $b \in M$, and (2) given any finite subset of elements of $M$ whose join exists in $P$, then that join is in fact in $M$. (We hasten to point out that "finite" subset means possibly empty subset.) The symbol $\varnothing$ will denote the empty set. Notice that $\varnothing$ has a join if and only if $P$ has a least element 0 . Thus $\varnothing$ is an ideal of $P$ if and only if $P$ has no zero element.

It is clear that the intersection of an arbitrary family of ideals of $P$ is an ideal of $P$. Also, the union of any chain of ideals is an ideal.

For any subset $X$ of $P$ the ideal generated by $X$ is the intersection of all ideals that contain $X$. It is denoted by $\mathscr{I}(X)$, and can be obtained as follows: $\mathscr{I}(X)=\bigcup\left\{A_{j} \mid j=1,2, \cdots\right\}$ where $A_{0}=X$ and $A_{j+1}=\{y \in P \mid$ ihere is a finite subset $F$ of $A_{j}$ whose join exists and exceeds $\left.y\right\}$.

Received by the editors December 16, 1970 and, in revised form, August 3, 1971.

AMS 1969 subject classifications. Primary 0650.

Key words and phrases. Ideal of a p.o. set, regular ideal, special ideal, strong finite join irreducibility, finite join irreducibility, freely generated, prime ideal, unique factorization p.o. set.

(c) American Mathematical Society 1972 
1.1 Proposition. The set of ideals of a p.o. set $P$ is a complete lattice whose meet operation is the intersection of sets and in which the join of the ideals $\left\{M_{i} \mid i \in I\right\}$ is $\mathscr{I}\left(\bigcup M_{i}\right)$.

(We denote this complete lattice by $\mathscr{P}$.)

An ideal $M$ of the p.o. set $P$ is a value of $x \in P$ if $x \notin M$ and $x$ is contained in any ideal that properly contains $M . M$ is regular if it is a value of some element of $P$; $\mathscr{R}$ will denote the p.o. set (under set inclusion) of regular ideals of $P$. An element $x \in P$ is special if it has only one value; if the ideal $M$ is the value of a special element that $M$ is also called special. If $x$ is special and $M$ is its value we say $x$ is special at $M$. (Note: each element of the p.o. set $P$ (except 0 if it exists) has at least one value; an easy application of Zorn's lemma gives an immediate proof.) The p.o. set of special ideals is denoted by $\mathscr{S}$.

We need a few more definitions: an ideal $N$ of $P$ is thoroughly prime if for every subset $A$ of $P$ whose meet exists and is in $N, N$ contains an of $A$. A dual o-ideal of the set $P$ is one which contains along with $x \in P$ all elements exceeding $x$. Finally, $x \in P$ is strongly finitely join irreducible (abbr. strongly f.j.i.) if, for every finite subset $F$ of $P$ whose join $\bigvee F$ exists and exceeds $x, x \leqq y$ for some $y \in F$.

We are now ready to state our main theorem (in part).

1.2 THEOREM. For a p.o. set $P$ the following are equivalent.

(1) The lattice $\mathscr{P}$ of ideals is completely distributive.

(2) Every regular ideal is special.

(3) Each $x \in P$ can be expressed uniquely as the join of a finite number of pairwise incomparable strongly $f$.j.i. elements.

For a lattice $L$ the above theorem translates as follows.

1.3 THEOREM. For a lattice $L$ the following are equivalent.

(1) The lattice $\mathscr{L}$ of ideals is completely distributive.

(2) Every regular ideal is special.

(3) Each element $x \in L$ can be written uniquely as a finite join of pairwise incomparable strongly $f$.j.i. elements.

(4) $L$ is distributive, and each $x \in L$ can be expressed uniquely as a finite join of pairwise incomparable f.j.i. elements.

REMARKS. (a) The term "completely distributive" is used to describe the validity of the most general distribution of meet over join.

(b) In the lattice $L$ an element $x$ is finitely join irreducible (abbr. f.j.i.) if $x=a \vee b$ implies $x=a$ or $x=b$.

(c) A note on the plan of the proof of Theorem 1.2: we shall demonstrate the equivalence of (2) and (3) in the next section. In $\S 3$ we will prove 
the equivalence of (1) and (2) by tying the situation to some work of Conrad in [4].

(d) For complete distributive lattices, Papert [5] has given a characterization of lattices having property (4) of Theorem 1.3.

Some remarks on notation and background material are in order here. For the basics in lattice theory the reader is referred to [1] and [2]. For subsets $A$ and $B$ of a set $X, A \backslash B$ will denote the usual set complement. The symbol $(\subset) \subseteq$ will be used for (proper) containment of sets. In a p.o. set $P$ we will write $a \| b$ when the elements $a$ and $b$ are incomparable.

The author wishes to thank the referee for many valuable suggestions, in particular the example following the proof of Corollary 3.1.2.

2. In the discussion below $P$ will be a p.o. set, and $\mathscr{P}$ its lattice of ideals. The first observation is a simple one; it is proved by an application of Zorn's lemma.

2.1 Proposition. If $M \in \mathscr{P}$ and $x \in P \backslash M$ there is some value of $x$ that contains $M$.

2.2 Proposition. Each ideal $M$ of $P$ is a meet of regular ideals.

Proof. If $M=P$ we write it as the meet of the empty family of regular ideals. If $M \subset P$ we take for each $x \notin M$ a value $M_{x}$ of $x$ containing $M . M$ is then the meet of all of these.

2.3 LEMMA. Given $x$ and $y$ in $P, x \leqq y$ if and only if every value of $x$ is contained in a value of $y$.

Proof. The necessity is clear. Conversely, suppose $x \neq y$; the ideal, $\mathscr{I}(y)=\{z \in P \mid z \leqq y\}$, misses $x$, so we can select a value $N$ of $x$ that contains $\mathscr{I}(y)$ and hence $y$ itself. $N$ can therefore not be contained in any values of $y$.

2.3.1 COROLlary. Two elements of a p.o. set coincide if and only if they have the same values.

2.4 Proposition. Let $M$ be a special value in $P$, and $x$ be special at $M$. Then $M=\{z \in P \mid z \geq x\}$. In particular, $M$ uniquely determines $x$.

Proof. Certainly if $z \geqq x$ then $z \notin M$. Conversely, if $z \notin M$ pick a value $N$ of $z$ containing $M$; since $M$ is the lone value of $x$ it follows from 2.3 that $x \leqq z$.

2.4.1 Corollary. An ideal $M$ of $P$ is special if and only if it is thoroughly prime and $\wedge P \backslash M$ exists.

Proof. Once again the necessity is clear. As for the sufficiency, suppose $M$ is thoroughly prime and $\wedge P \backslash M$ exists. Let $x=\wedge P \backslash M$; we claim that $M=\{z \in P \mid z \geq x\}$. Certainly $x \notin M$ because $M$ is thoroughly 
prime, so $M$ is a subset of the set in question; on the other hand if $z \notin M$ then, by definition of $x, z \geqq x$. It is evident then that $x$ is special at $M$.

2.5 Proposition. The following are equivalent for $x \in P$.

(i) $x$ is special;

(ii) $x$ is strongly f.j.i.;

(iii) the set $M(x)=\{z \in P \mid z \geq x\}$ is an ideal of $P$.

If $P$ is a distributive lattice the above are equivalent to

(iv) $x$ is f.j.i.

Proof. Clearly (ii) and (iii) are equivalent. (i) $\rightarrow$ (iii) is immediate from 2.4; on the other hand if (iii) holds then $M(x)$ is plainly the largest ideal missing $x$, and thus $x$ is special.

If $P$ is a distributive lattice it is an easy exercise to show (ii) and (iv) are equivalent.

EXAMPLE. Let $L$ be the lattice $\{0, a, b, c, 1\}$ with $0<x<1$ for $x=$ $a, b, c$ and $a \vee b=a \vee c=b \vee c=1$ while $a \wedge b=a \wedge c=b \wedge c=0$. Then $L$ has no special elements, yet $a, b, c$ are all f.j.i.; it is, however, not a distributive lattice. We shall return to this example later.

2.5.1 CoRollary. The map that assigns to each special element of $P$ its only value is an o-isomorphism from the p.o. set of strongly f.j.i. elements onto $\mathscr{S}$.

For $M \in \mathscr{R}$ let $\hat{M}=\{a \in P \mid$ each value of $a$ is contained in $M\}$. It is not hard to show that $\hat{M}$ is always an ideal of $P$; if in addition $M$ is special this is trivial for then $\hat{M}$ is simply the ideal generated by $x$, where $x$ is special at $M$.

2.6 LEMMA. If all the values of $x \in P$ are special then $x$ has finitely many values.

Proof. Let $\left\{C_{\alpha} \mid \alpha \in A\right\}$ be the set of values of $x$, and $C=\bigvee \hat{C}_{\alpha}$; pick $c_{\alpha}$ special at $C_{x}$. We claim $x \in C$; otherwise there is a value $C_{\beta}$ of $x$ containing $C$. But $c_{\beta} \in \hat{C}_{\beta} \subseteq C \subseteq C_{\beta}$, a contradiction. But then it follows (after a suitable well ordering of $A$ ) that $x \in \hat{C}_{1} \vee \hat{C}_{2} \vee \cdots \vee \hat{C}_{n}{ }^{1}$. Now select a value $Q$ of $x$; if each $c_{i} \in Q$ then $\hat{C}_{1} \vee \hat{C}_{2} \vee \cdots \vee \hat{C}_{n} \subseteq Q$ (since the left-hand side of the inequality is the ideal generated by $c_{1}, c_{2}, \cdots, c_{n}$ ). Consequently some $c_{j} \notin Q$ and so $Q \subseteq C_{j}$ since $c_{j}$ is special at $C_{j}$. In fact equality holds since both are values of $x$. What we have actually shown is that the $C_{i}(1 \leqq i \leqq n)$ are the only values of $x$, and the lemma is proved.

\footnotetext{
${ }^{1}$ The closure operator is algebraic; that is, if $a \in \mathscr{I}(X)$ then $a \in \mathscr{I}(Y)$ where $Y$ is a finite subset of $X$. This follows from our observation that $\mathscr{P}$ is inductive, and Theorem 1.2, on p. 45 in P. M. Cohn's Universal algebra, Harper \& Row, New York, 1965.
} 
We can now prove the equivalence of (2) and (3) in Theorem 1.2: let $x \in P$ and suppose all its values are special. According to 2.6 it has a finite number of values $M_{1}, \cdots, M_{k}$. Let $x_{i}$ be special at $M_{i}$; then $x \geqq x_{i}$ by 2.3 . Further if $y \geqq x_{i}(1 \leqq i \leqq n)$ then again by 2.3 each $M_{i}$ is contained in some value of $y$; this says precisely that $y \geqq x$. We conclude then that $x=$ $x_{1} \vee \cdots \vee x_{n}$. Since $M_{i} \| M_{j}$ if $i \neq j$, we have $x_{i} \| x_{j}$; the uniqueness of this representation is a consequence of Corollary 2.3.1.

We have shown that $(2) \rightarrow(3)$; the converse is now straightforward, and is left to the reader.

3. To complete the proof of Theorem 1.2 we need some results of Conrad in [4]. Let $L$ be a lattice; an element $x \in L$ is meet irreducible if $x<a_{\lambda}$, for all $\lambda$, implies $x<\bigwedge a_{\lambda}$ provided the indicated meet exists. $L$ is generated by the subset $S$ of meet irreducible elements if (1) each $a \in L$ is the meet of a dual $o$-ideal in $S$, and (2) every dual $o$-ideal in $S$ has a meet in $L$. If in addition $\bigwedge N_{1}=\bigwedge N_{2}$ implies that $N_{1}=N_{2}$ for each pair of dual $o$-ideals of $S$, we say' $S$ freely generates $L$. We can now state one of the main theorems in [4].

THEOREM (CONRAD). If $L$ is a lattice that is generated by its subset $S$ of meet irreducible elements, then the following are equivalent:

(a) $S$ freely generates $L$;

(b) $L$ is completely distributive;

(c) $b \vee\left(\bigwedge a_{\lambda}\right)=\bigwedge\left(b \vee a_{\lambda}\right)$, for all $a_{\lambda}, b \in S(\lambda \in \Lambda)$.

We list some further results from [4]. Below, let $L$ be a lattice generated by the subset $S$ of meet irreducibles. For $a \in L$ let $D(a)=\{x \in S \mid x \geqq a\}$; plainly, $D(a)$ is a dual $o$-ideal in $S$.

1. For each subset $\left\{a_{\lambda} \mid \lambda \in \Lambda\right\}$ of $L$,

$$
\wedge a_{\lambda}=\wedge\left\{x \mid x \in \cup D\left(a_{\lambda}\right)\right\}, \quad \vee a_{\lambda}=\wedge\left\{y \mid y \in \cap D\left(a_{\lambda}\right)\right\} .
$$

In particular $L$ is complete (2.4 of [4]).

2. $D\left(\bigvee a_{\lambda}\right)=\bigcap D\left(a_{\lambda}\right)$ and $D\left(\bigwedge a_{\lambda}\right) \supseteq \bigcup D\left(a_{\lambda}\right)$, and equality holds if and only if $S$ freely generates $L$ (2.4 of [4] and corollary).

3. If $D(a \wedge b)=D(a) \cup D(b)$, for all $a, b \in L$, then

$$
x \wedge\left(\bigvee y_{\gamma}\right)=\bigvee\left(x \wedge y_{\gamma}\right), \quad \gamma \in \Gamma
$$

in particular, $L$ is distributive (2.5 in [4]).

We now return to the situation of $\S 2: P$ is a p.o. set and $\mathscr{P}$ is the lattice of ideals. Birkhoff and Frink proved (Corollary 1 to Theorem 8 in [3]) that in $\mathscr{P}$ the meet irreducibles are precisely the regular ideals. (Note: $P$ itself is not meet irreducible!) $\mathscr{P}$ is then generated by $\mathscr{R}$ in Conrad's sense; see 2.2. To the discussion we add 
3.1 THEOREM. $\mathscr{R}$ freely generates $\mathscr{P}$ if and only if each regular ideal is special.

Before proving this we record a corollary:

3.1.1 Corollary. If $P$ is a p.o. set and $\mathscr{P}$ is its lattice of ideals, the following statements are equivalent.

(1) $\mathscr{R}$ freely generates $\mathscr{P}$.

(2) $\mathscr{P}$ is completely distributive.

(3) Each regular ideal is special.

(4) Each $x \in P$ can be written uniquely as a finite join of pairwise incomparable strongly f.j.i. elements. ${ }^{2}$

If $P$ is a lattice the above statements are equivalent to

(5) $P$ is distributive, and each $x \in P$ can be expressed uniquely as a finite join of pairwise incomparable f.j.i. elements.

ProOF OF THE COROLlary. We already know that the first four statements are equivalent. If $P$ is a lattice and (2) holds then $P$ must be distributive, for $P$ is embedded in $\mathscr{P}$ as a sublattice by taking $a \in P$ to $\mathscr{I}(a)$. From Proposition 2.5 it is then clear why (5) is equivalent to the rest. At this point the proofs of both Theorem 1.2 and Theorem 1.3 are completed (modulo the proof of Theorem 3.1).

Proof of 3.1. Suppose $M \in \mathscr{R}$ is not special. Let $\mathscr{V}_{1}=\{D \in \mathscr{R} \mid D \nsubseteq M\}$; this is a dual $o$-ideal of $\mathscr{R}$. Let $\mathscr{N}=\mathscr{N}_{1} \cup\{M\}$; clearly $\cap \mathscr{r} \subseteq \cap \mathscr{N}_{1}$. If $x \in \cap \mathscr{V}_{1}$ and $Q$ is a value of $x$ then $Q \subseteq M . Q \neq M$ for otherwise $x$ would be special at $M$, contrary to assumption. Thus $Q \subset M$ and hence $x \in \cap \mathscr{V}$; that is $\cap \mathscr{V}=\cap \mathscr{H}_{1}$, which says that $\mathscr{R}$ does not generate $\mathscr{P}$ freely.

Conversely, if $\mathscr{R}$ does not generate $\mathscr{P}$ freely there is a dual $o$-ideal $\mathscr{N}=\left\{N_{\sigma} \mid \sigma \in \Sigma\right\}$ and $M \in \mathscr{R} \mid \mathcal{V}$ such that $\bigcap N_{\sigma} \subseteq M$; thus $N_{\sigma} \nsubseteq M$ for all $\sigma \in \Sigma$. Let $\mathscr{H}=\{D \in \mathscr{R} \mid D \subseteq M\}$; then $\mathscr{V} \subseteq \mathscr{H}$ which says that $\cap \mathscr{H} \subseteq$ $\cap \mathscr{V} \subseteq M$. If $M$ were special we could pick $x \in P$ special at $M$; but then $x \in \cap \mathscr{M} \subseteq M$, a contradiction. It follows that $M$ is not special.

We should not fail to observe here that the proofs of Lemma 2.6 and Theorem 3.1 were adapted, and very smoothly at that, from proofs of corresponding results in [4].

A p.o. set $P$ satisfying condition (3) in Theorem 1.2 is called a unique factorization p.o. set. A lattice with this property is termed a unique factorization lattice.

\footnotetext{
${ }^{2}$ Each of the conditions of Corollary 3.1 .1 is equivalent to the following: $\not{P}$ is isomorphic to the full ring of dual $o$-ideals of $\mathscr{K}$, the isomorphism being realized by the map that assigns to an ideal $M$ the dual $o$-ideal $D(M)$.
} 
3.1.2 COROllary. In a unique factorization p.o. set every nonzero element has a finite number of values.

Proof. Using Corollary 3.1.1 this is merely a restatement of Lemma 2.6.

The converse of the above corollary is false, as pointed out by the following example, due to the referee. Let $L$ be the set of all points in the real plane whose coordinates $(x, y)$ both satisfy $0<x, y<1$. The lattice ordering is the coordinate-wise ordering, and $L$ is in fact a distributive lattice. It is not hard to see that no f.j.i. elements exist in $L$, yet every element has precisely two values: if $(x, y) \in L$ is given then

$$
C_{x}=\{(a, b) \in L \mid a<x\} \text { and } C_{y}=\{(a, b) \in L \mid b<y\}
$$

are the two values of $(x, y)$.

The example following Proposition 2.5 suggests how to construct a lattice with no special elements, in which all elements have a prescribed number of values, but also having the property that every element except 0 and 1 is f.j.i. (and 1 is the join of two f.j.i. elements). Let $\eta$ be a cardinal number and $H$ be a set of that cardinality. Define $L$ to be $H \cup\{0\} \cup\{1\}$ where (a) $0<h<1$, for all $h \in H$, and (b) $H$ is trivially ordered.

Finally, we wish to point out the connection between regular and prime ideals, at least in the setting of distributive lattices. An ideal $N$ of a p.o. set $P$ is prime if the set-theoretic complement of $N$ is an ideal in the dual order of $P$; equivalently if for every finite subset $F$ of $P$ having a meet $\bigwedge F, \wedge F \in M$ implies that $M \cap F \neq \varnothing$.

3.2 Proposition. Suppose $P$ is a p.o. set, and consider the following statements $(M$ is an ideal of $P)$ :

(1) $M \supseteq C_{1} \cap C_{2}$ implies $M \supseteq C_{1}$ or $M \supseteq C_{2}$, for all $C_{i} \in \mathscr{P}(i=1,2)$.

(2) $M$ is finitely meet irreducible in $\mathscr{P}$.

(3) $M$ is a prime ideal of $P$.

(1) implies (2); if $P$ is a lattice (3) implies (1). If $P$ is a distributive lattice the three statements are equivalent.

Proof. (1) says that $M$ is strongly finitely meet irreducible in $\mathscr{P}$; so (1) clearly implies (2). If $P$ is a lattice and (3) holds, suppose $M \supseteq C_{1} \cap C_{2}$ with $C_{i} \in \mathscr{P}(i=1,2)$. If $M \notin C_{1}$ select $a \in C_{1} \backslash M$; for each $b \in C_{2}, a \wedge b \in C_{1} \cap$ $C_{2} \subseteq M$, from which we may conclude that $b \in M$ since $M$ is prime. Thus $C_{2} \subseteq M$, and we have proved (3) $\rightarrow(1)$.

Finally, if $P$ is distributive and (2) holds we let, for each $x \in P,(M, x)$ denote the ideal generated by $M$ and $x$. Then it is easy to check that

(a) $(M, x)=\left\{m \vee x^{\prime} \mid m \in M\right.$ and $\left.x^{\prime} \leqq x\right\}$, and

(b) $(M, x \wedge y)=(M, x) \cap(M, y)$, for all $x, y \in P$. 
If $x$ and $y$ are not in $M$ then $(M, x)$ and $(M, y)$ both contain $M$ properly. By meet irreducibility, and in view of (b), $(M, x \wedge y) \supset M$, and so $x \wedge y \notin M$. It follows that $M$ is prime.

If we consider the trivially ordered set $\{1,2,3\}$, the subset $\{1\}$ is prime but not meet irreducible. ${ }^{3}$ On the other hand if $L$ is the lattice in the example following Proposition 2.5 the ideal $\{0, a\}$ is not prime (for $b \wedge c=0)$, but it is meet irreducible. Generally speaking then, 3.2 tells us the most we can expect from the relationship between meet irreducibility of an ideal and primeness.

3.2.1 COROLLARY. In a distributive lattice $L$

(a) each regular ideal is prime.

(b) $D\left(C_{1} \cap C_{2}\right)=D\left(C_{1}\right) \cup D\left(C_{2}\right)$, where $D(C)=\{M \in \mathscr{R} \mid M \supseteq C\}, C \subseteq \mathscr{L}$.

In particular then we obtain, in view of Conrad's results of [4] that the lattice $\mathscr{L}$ is distributive. Moreover, finite meets distribute over arbitrary joins; since $\mathscr{L}$ is complete it is a so-called Brouwerian lattice. This is a well-known result of M. H. Stone [2, p. 129, Theorem 25].

\section{BIBLIOGRAPHY}

1. J. C. Abbott, Sets, lattices, and Boolean algebras, Allyn \& Bacon, Boston, Mass., 1969. MR 39 \#4052.

2. G. Birkhoff, Lattice theory, 3rd ed., Amer. Math. Soc. Colloq. Publ., vol. 25, Amer. Math. Soc., Providence, R.I., 1967. MR 37 \#2638.

3. G. Birkhoff and O. Frink, Representations of lattices by sets, Trans. Amer. Math. Soc. 64 (1948), 299-316. MR 10, 279.

4. P. Conrad, The lattice of all convex l-subgroups of a lattice-ordered group, Czechoslovak Math. J. 15 (90) (1965), 101-123. MR 30 \#3926.

5. S. Papert, Which distributive lattices are lattices of closed sets?, Proc. Cambridge Philos. Soc. 55 (1959), 172-176. MR 21 \#3354.

Department of Mathematics, University of Florida, Gainesville, Florida 32601 .

${ }^{3}$ Notice that this trivially ordered set satisfies the conditions of Theorem 1.2. 